\title{
Septic arthritis of the hip joint caused by Klebsiella pneumoniae: a case report
}

\author{
Jeong-Bo Moon, Jun-Hwan Lee, Byung-Ju Ryu \\ Department of Physical Medicine and Rehabilitation, Sahmyook Medical Center, Seoul, Korea
}

\begin{abstract}
Klebsiella pneumoniae is an uncommon cause of septic arthritis in adults. However, late detection can cause serious complications, including joint destruction and immobility. The purpose of this study was to report a case of successfully treated septic arthritis of the hip joint (SAHJ) caused by K. pneumoniae. A 49-year-old female patient presented to our hospital with fever and progressive severe pain in the right hip area. Although there was no abnormality on plain radiographs, ultrasonography revealed diffuse swelling of the right hip joint. Under ultrasonography guidance, the hip joint fluid was aspirated, and Gram staining and culturing were performed. The patient's pain was significantly reduced after the joint aspiration. The Gram staining and culturing revealed gram-negative bacilli, which were subsequently identified as $K$. pneumoniae. According to the results, systemic intravenous antibiotic (ceftriaxone) was administered without complications, and the patient was discharged on oral antibiotic (ciprofloxacin). Clinical cases of septic arthritis of the knee or sacroiliac joint have been occasionally reported in adults, but cases of SAHJ are rare. Moreover, $K$. pneumonia-induced SAHJ has not been reported to date. Therefore, we report this very rare case and its successful treatment.
\end{abstract}

Keywords: Hip; Infectious; Klebsiella pneumoniae; Septic arthritis

\section{Introduction}

Septic arthritis is a bacterial, mycobacterial, or fungal joint infection. It is associated with significant morbidity and mortality $[1,2]$. Clinical cases of septic arthritis of the knee joint have occasionally been reported. However, cases of septic arthritis of the hip joint (SAHJ) are rarely reported, and all previous reports involved immunocompromised patients [2,3]. SAHJ presents a diagnostic challenge because it mimics other musculoskeletal conditions, such as degenerative hip joint disease or tendonitis (Table 1) [4]. Septic arthritis is most commonly caused by gram-positive bacteria, including Staphylococcus aureus, Staphylococcus epidermidis, and streptococci [1]. Klebsiella pneumoniae, a gram-negative bacillus of the family Enterobacteriaceae, is an uncommon cause of septic arthritis in adults [3]. Herein, we describe a case of acute SAHJ caused by $K$. pneumoniae in a patient with no primary lesions or underlying medical conditions. Informed consent for publication of the patient's clinical details and clinical images was obtained from the patient.

\section{Case}

Ethical statements: This study was exempted from review by the Institutional Review Board (IRB) of Sahmyook Medical Center (IRB No: 116286-202111-HR-02). Written informed consent was obtained from the patients to participate in the study.

Received: November 16, 2021 • Revised: November 19, 2021 • Accepted: November 22, 2021

Corresponding author: Byung-Ju Ryu, MD

Department of Physical Medicine and Rehabilitation, Sahmyook Medical Center, 82 Mangu-ro, Dongdaemun-gu, Seoul 02500, Korea

Tel: 82-2-2210-3133 • Fax: 82-2-2210-3133•E-mail: btjrbj@gmail.com 
Table 1. Differential diagnosis of septic arthritis of the hip joint and other musculoskeletal disorders in adults

\begin{tabular}{|c|c|}
\hline Differential diagnosis & Clinical feature \\
\hline Lumbar radiculopathy & Pain in the posterior buttock or radiating pain down the thigh, which may be a burning sensation \\
\hline Sacroiliac joint disease & Pain in the posterior buttock \\
\hline Piriformis syndrome & $\begin{array}{l}\text { Pain in the posterior buttock with activity, sciatic pain radiating down the leg as a result of nerve compression, ten- } \\
\text { derness over the piriformis tendon and muscle }\end{array}$ \\
\hline Osteonecrosis & Pain in the hip or groin, gradually progresses \\
\hline Occult femoral neck fracture & $\begin{array}{l}\text { Pain in the hip or groin, can be traumatic, associated with accompanying injury, or atraumatic, resulting in a stress } \\
\text { fracture }\end{array}$ \\
\hline Meralgia paresthetica & Pain in the hip or groin, radiating down the thigh's anterolateral side \\
\hline Psoas tendinopathy & Pain in the groin, associated with resisted hip flexion \\
\hline Trochanteric bursitis & Pain localized to greater trochanter, palpable tenderness, minimal pain in the groin \\
\hline Abductor tendon tear & Pain localized to greater trochanter, minimal pain in the groin \\
\hline Neurogenic claudication & $\begin{array}{l}\text { Back pain is more severe than leg pain, radiating down the leg; it is worse when standing upright and improves when } \\
\text { seated bent forward }\end{array}$ \\
\hline Vascular claudication & Leg pain is more severe than back pain, is aggravated by walking and any movement, and improves with rest \\
\hline Iliac avulsion fracture & Pain in the hip following trauma or unexpected muscle activation \\
\hline Traumatic synovitis, hemarthrosis & Recent trauma history \\
\hline Gout & Pain caused by polyarthralgia, aggravated by particular foods \\
\hline Pseudogout & Pain caused by polyarthralgia, more prevalent among those who are elderly \\
\hline Rheumatoid arthritis flare & Pain caused by polyarthralgia, patient may be on immunosuppressive medication \\
\hline Osteoarthritis exacerbation & Pain that has recently worsened as a result of increased activity \\
\hline
\end{tabular}

A 49-year-old housewife presented to our rehabilitation department with a 12-hour history of sudden-onset, progressively worsening pain in the right hip area, with concomitant fever that developed 2 hours after pain onset. She complained of severe pain with a numerical rating scale (NRS) score of 8 when bending her right hip and was unable to ambulate because of severe pain when bearing weight on her right leg. She had no comorbidities or history of trauma. She mentioned high levels of stress for personal reasons, which had persisted for 6 months.

Upon arrival at the hospital, her vital signs were as follows: body temperature, $37.8^{\circ} \mathrm{C}$; pulse rate, 93 beats $/ \mathrm{min}$; respiratory rate, 20 breaths/min; and blood pressure, 128/80 mmHg. Physical examination revealed tenderness of the right medial thigh and painful limitation of movement, especially internal rotation. Laboratory tests revealed a white blood cell (WBC) count of $14,200 / \mu \mathrm{L}$ with neutrophilic predominance (87.6\%), a hemoglobin level of $13.3 \mathrm{~g} /$ $\mathrm{dL}$, and a platelet count of $295,000 / \mu \mathrm{L}$. Plain radiographs showed no abnormalities, except for mild degenerative changes in both hip joints. Ultrasonography revealed diffuse swelling of the right hip joint (Fig. 1A). Under ultrasonographic guidance, $12 \mathrm{~mL}$ of yellow, cloudy, moderately viscous fluid was aspirated from the right hip joint, and joint fluid analysis, Gram staining, and culturing were performed (Fig. 1B). The patient's hip pain was significantly reduced ( 4 on the NRS) after hip joint fluid aspiration; she was able to walk but had general myalgia. Since the patient's clinical signs and symptoms had improved rapidly, and the results of Gram staining and culturing were not yet available, she was discharged with an outpatient appointment scheduled for 4 days thereafter.

Four days later, the patient was admitted to the hospital. Laboratory tests showed a WBC count of $6,229 / \mu \mathrm{L}$ with neutrophilic predominance $(67.5 \%)$, a hemoglobin level of $14.1 \mathrm{~g} / \mathrm{dL}$, and a platelet count of $356,000 / \mu \mathrm{L}$. The joint fluid showed a WBC count of $8,979 / \mathrm{mm}^{3}$ with neutrophilic predominance $(78 \%)$ and a red blood cell count of $1,000 / \mathrm{mm}^{3}$; septic arthritis was suspected. The Gram staining and culturing revealed gram-negative bacilli, which were subsequently identified as K. pneumoniae. Susceptibility test results indicated sensitivity to all antibiotics, including cefepime, meropenem, piperacillin/tazobactam, ceftazidime/avibactam, ceftriaxone, colistin, ertapenem, tigecycline, amikacin, ampicillin/ sulbactam, cefazolin, ceftazidime, ciprofloxacin, gentamicin, imipenem, and trimethoprim/sulfamethoxazole, but not ampicillin alone. The patient was hospitalized for antibiotic treatment and underwent further evaluation. Ceftriaxone ( $2 \mathrm{~g}$, once a day) was administered intravenously for 3 days. A whole-body bone scan was performed to identify complications of SAHJ and revealed no abnormal uptake anywhere in the body, including the right hip joint (Fig. 2). Computed tomography of the abdomen and pelvis to investigate primary lesions of $K$. pneumoniae infection showed no specific abnormalities except for a 6-cm, unilocular, cystic lesion at the right adnexa suspected of being a benign ovarian cyst or a mucinous cystic neoplasm. The patient had no underlying medical conditions that could lead to an immunocompromised state, 

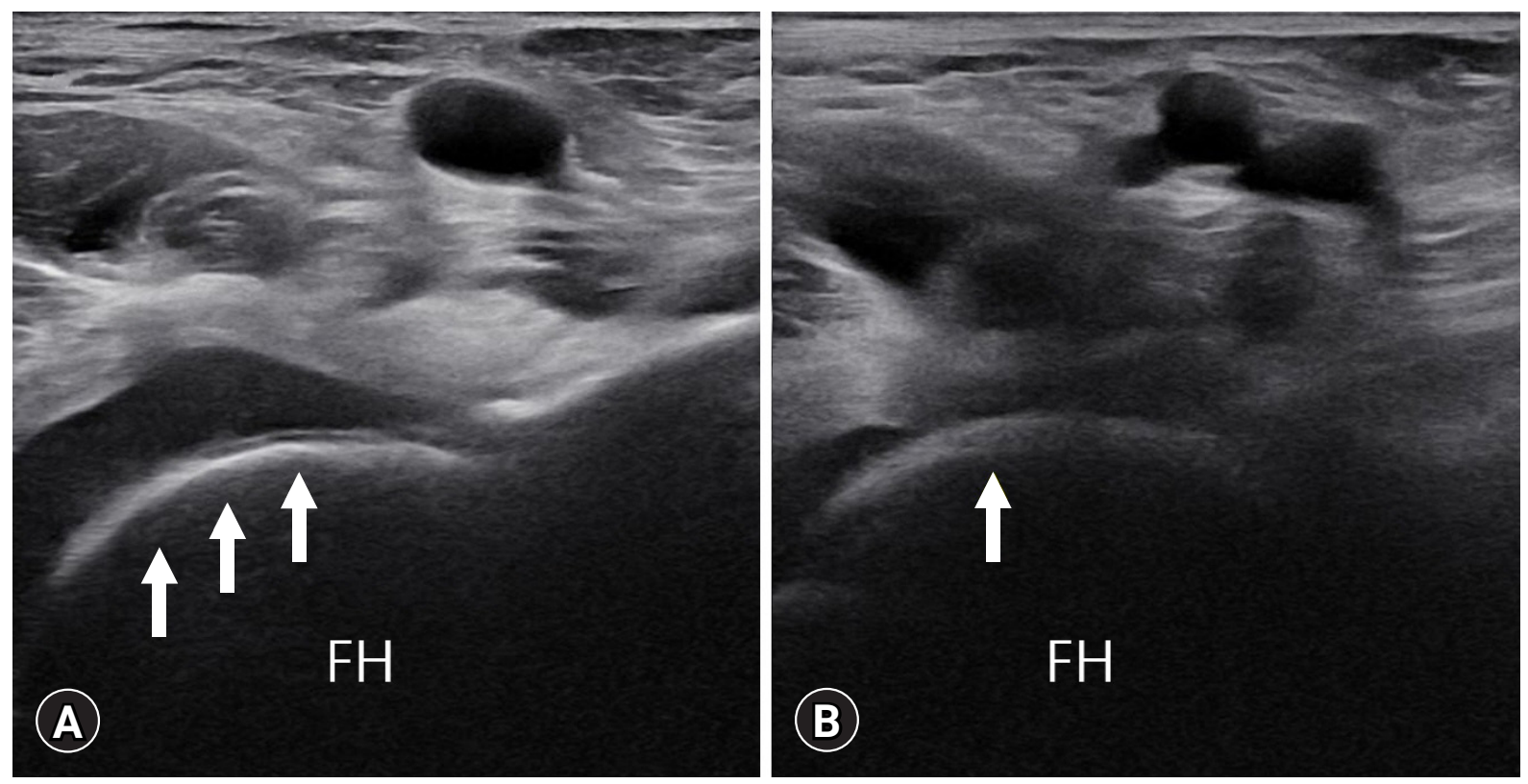

Fig. 1. Pre- and post-aspiration ultrasonography images of the right hip joint. (A) Mild fluid collection and diffuse swelling of the right hip joint (arrows) are seen in the pre-aspiration image. (B) A decrease in the fluid collected in the right hip joint (arrow) is seen in the post-aspiration image. $\mathrm{FH}$, femoral head.

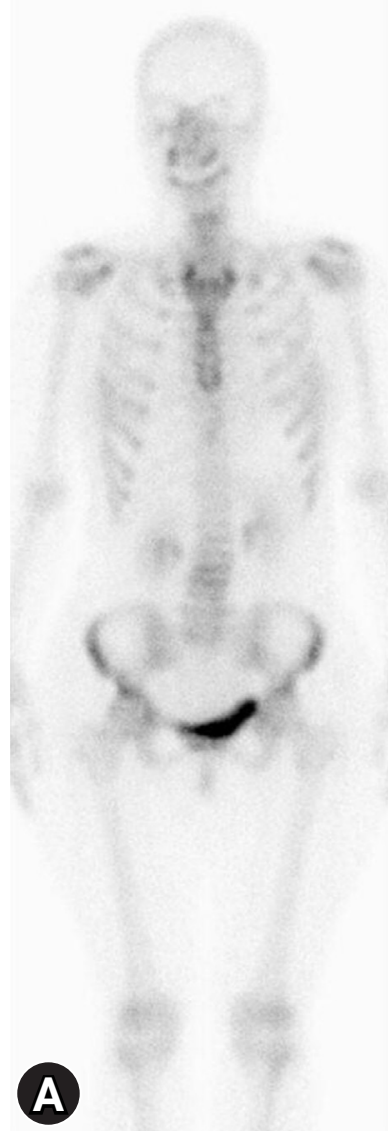

ANT Alpha: 30\% 1423K

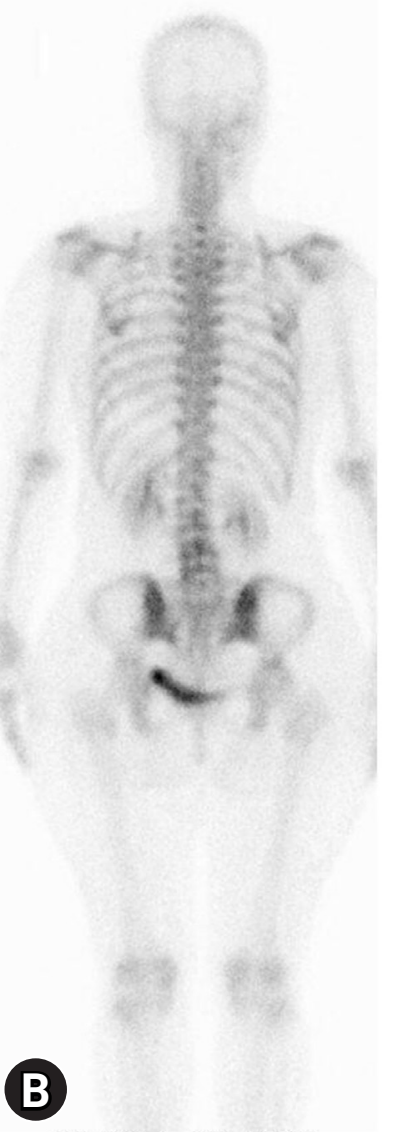

POST Alpha: $30 \%$ 1235K

Fig. 2. (A) Anterior and (B) posterior views of a whole-body bone scan. No abnormal findings such as hot or cold spots are observed throughout the body including the right hip joint.

such as diabetes, chronic renal failure, malignancy, alcoholism, or acquired immunodeficiency syndrome. She was uneventfully discharged 3 days later while receiving oral antibiotics (ciprofloxacin, $500 \mathrm{mg}$, twice daily for 7 days). At her 6-month follow-up, she complained of no hip pain and showed no specific features on review of systems.

\section{Discussion}

To the best of our knowledge, this is the first report of a case of acute SAHJ caused by K. pneumoniae in a patient with no primary lesion or underlying disease.

It is difficult to estimate the incidence of SAHJ in adults, which has mostly been reported in case reports [1]. Numerous prior studies have dealt with all sites of septic arthritis collectively, despite significant variance in clinical manifestations among joints. The incidence of septic arthritis in the general population of industrialized countries is 2 to 6 cases per 100,000 people [5] and 5 to 12 cases per 100,000 children [6,7]. However, the overall incidence appears to be increasing, and this increase is associated with an aging population, an increasing number of invasive procedures being performed, and the increased use of immunosuppressive therapies $[6,8]$.

Organisms that cause septic arthritis differ according to patient age. The most common causative agent of bacterial arthritis in adults is S. aureus, with an incidence between 37\% and 67\% [9]. 
Following this, $9 \%$ to $20 \%$ of bacterial arthritis is caused by gramnegative bacteria such as Escherichia coli [10]. In children, the most frequently observed causal organisms are methicillin-sensitive $S$. aureus, Streptococcus pneumoniae, and Haemophilus influenzae [11]. $K$. pneumoniae rarely causes septic arthritis in all age groups. It is known to cause septic arthritis as a secondary infection through hematogenous transmission after primary infections, such as liver abscesses, pneumonia, and urinary tract infections [12]. K. pneumoniae infection of other organs rarely occurs in the absence of a liver abscess, although it may occur in special clinical situations, including trauma, intravenous drug abuse, and immunosuppression following organ transplant or human immunodeficiency virus infection $[2,12,13]$. In this case, a 49-year-old woman with no known underlying disease developed SAHJ caused by K. pneumoniae in the absence of any primary lesion, such as a liver abscess.

Magnetic resonance imaging or surgical intervention was not performed to determine whether the bones and muscles surrounding the hip joint were affected. The reason for this decision was that the patient's clinical symptoms of fever and hip pain improved rapidly after hip joint fluid aspiration, and the subsequent laboratory test after 4 days also improved. In addition, a whole-body bone scan revealed no abnormal uptake throughout the body. Based on these findings, we concluded that an appropriate antibiotic was sufficient to control her SAHJ.

Generally, septic arthritis is treated with drainage, cleaning of the joint abscess, and systemic antibiotic therapy based on the results of susceptibility testing. Appropriate drainage is essential for the management of purulent infections in enclosed spaces. There have been prior cases of children undergoing high-volume lavage and irrigation, and cases of adults undergoing open arthrotomy and ultrasonography-guided irrigation. Arthroscopic lavage and debridement can be performed to drain and clean intraarticular abscesses [14]. Goldenberg et al. [15] compared needle aspiration with surgical intervention, and no evidence was available to recommend one treatment strategy over the other. In our case, the hip joint fluid was sufficiently aspirated under ultrasonographic guidance, and ceftriaxone-susceptible K. pneumoniae was identified by Gram staining and culture of the joint fluid. According to the guidelines for treating septic arthritis, it is important to immediately remove the purulent material from inside the joint and administer systemic antibiotics that are effective against the causative organism [16]. Accordingly, in this case, immediate pus aspiration and antibiotic treatment based on the results of susceptibility testing were sufficient to treat the disease and resulted in complete recovery without sequelae, such as residual impairment due to hip joint destruction.

Considering the pathophysiology of joint destruction in septic arthritis, it is known that the synovium in the joint is an abundantly vascularized structure without a restricting base plate, and bacteria have easy access [17]. Extracellular virulence agents produced by bacteria are critical for the progression of erosive joint destruction associated with septic arthritis [18]. As the infection progresses, an effusion forms in the joint, increasing the intraarticular pressure and preventing blood supply to the joint. The reported fatality rates for monoarticular septic arthritis vary by report, but are estimated to be $\sim 11 \%$ [1]. Patients with acute septic arthritis often present with fatigue, erythema, edema, discomfort, and limited range of motion in a single joint over a period of 1 to 2 weeks [19].

Septic arthritis in children under the age of 2 years may go undiagnosed due to the absence of typical indications of infection; thus, caution should be exercised when examining them. Anxiety, irritability, tachycardia, and anemia are all possible clinical indications and symptoms [20].

We report a very rare case of SAHJ caused by K. pneumoniae that developed in a relatively young patient with no medical history. SAHJ should be considered during the differential diagnosis of patients with acute hip pain despite the absence of plain radiographic abnormalities.

\section{Notes}

\section{Conflicts of interest}

No potential conflict of interest relevant to this article was reported.

\section{Funding}

None.

\section{Author contributions}

Conceptualization: BJR; Visualization: JBM, JHL; Writing-original draft: JBM; Writing-review \& editing: BJR.

\section{ORCID}

Jeong-Bo Moon, https://orcid.org/0000-0002-6689-5826

Jun-Hwan Lee, https://orcid.org/0000-0002-3180-2300

Byung-Ju Ryu, https://orcid.org/0000-0003-0109-9885

\section{References}

1. Mathews CJ, Weston VC, Jones A, Field M, Coakley G. Bacterial septic arthritis in adults. Lancet 2010;375:846-55.

2. Tseng CC, Wu CD, Lin WT, Chan HT, Chen PY. Acute septic arthritis caused by Klebsiella pneumoniae. Formos J Musculoskelet Disord 2013;4:51-2.

3. Schelenz S, Bramham K, Goldsmith D. Septic arthritis due to 
extended spectrum beta lactamase producing Klebsiella pneumoniae. Joint Bone Spine 2007;74:275-8.

4. Chee YC, Lim CH. Klebsiella pneumoniae sacroiliac septic arthritis: first case report. IDCases 2018; 14:e00459.

5. Kaandorp CJ, Van Schaardenburg D, Krijnen P, Habbema JD, van de Laar MA. Risk factors for septic arthritis in patients with joint disease: a prospective study. Arthritis Rheum 1995;38: 1819-25.

6. Nade S. Septic arthritis. Best Pract Res Clin Rheumatol 2003; 17:183-200.

7. Frank G, Mahoney HM, Eppes SC. Musculoskeletal infections in children. Pediatr Clin North Am 2005;52:1083-106.

8. Geirsson AJ, Statkevicius S, Víkingsson A. Septic arthritis in Iceland 1990-2002: increasing incidence due to iatrogenic infections. Ann Rheum Dis 2008;67:638-43.

9. Ohl CA, Forster D. Infectious arthritis of native joints. In: Mandell GL, Bennett JE, Dolin R, editors. Mandell, Douglas, and Bennett's principles and practice of infectious diseases. 6th ed. Philadelphia: Elsevier; 2005. p. 1302-17.

10. Chew LC. Septic monoarthritis and osteomyelitis in an elderly man following Klebsiella pneumoniae genitourinary infection: case report. Ann Acad Med Singap 2006;35:100-3.

11. Barton LL, Dunkle LM, Habib FH. Septic arthritis in childhood: a 13-year review. Am J Dis Child 1987;141:898-900.

12. Lee HC, Chuang YC, Yu WL, Lee NY, Chang CM, Ko NY, et al. Clinical implications of hypermucoviscosity phenotype in Klebsiella pneumoniae isolates: association with invasive syn- drome in patients with community-acquired bacteraemia. J Intern Med 2006;259:606-14.

13. Hsieh PF, Lin TL, Lee CZ, Tsai SF, Wang JT. Serum-induced iron-acquisition systems and TonB contribute to virulence in Klebsiella pneumoniae causing primary pyogenic liver abscess. J Infect Dis 2008;197:1717-27.

14. Stutz G, Kuster MS, Kleinstück F, Gächter A. Arthroscopic management of septic arthritis: stages of infection and results. Knee Surg Sports Traumatol Arthrosc 2000;8:270-4.

15. Goldenberg DL, Brandt KD, Cohen AS, Cathcart ES. Treatment of septic arthritis: comparison of needle aspiration and surgery as initial modes of joint drainage. Arthritis Rheum 1975;18:83-90.

16. Coakley G, Mathews C, Field M, Jones A, Kingsley G, Walker D, et al. BSR \& BHPR, BOA, RCGP and BSAC guidelines for management of the hot swollen joint in adults. Rheumatology (Oxford) 2006;45:1039-41.

17. Goldenberg DL. Septic arthritis. Lancet 1998;351:197-202.

18. Abdelnour A, Arvidson S, Bremell T, Rydén C, Tarkowski A. The accessory gene regulator (agr) controls Staphylococcus aureus virulence in a murine arthritis model. Infect Immun 1993; 61:3879-85.

19. Shirtliff ME, Mader JT. Acute septic arthritis. Clin Microbiol Rev 2002;15:527-44.

20. Morgan DS, Fisher D, Merianos A, Currie BJ. An 18 year clinical review of septic arthritis from tropical Australia. Epidemiol Infect 1996;117:423-8. 